\title{
A Bijective Proof of the Alladi-Andrews-Gordon Partition Theorem
}

\author{
James J.Y. Zhao \\ Center for Applied Mathematics \\ Tianjin University, Tianjin 300072, P. R. China \\ jjyzhao@tju.edu.cn
}

Submitted: Jul 28, 2014; Accepted: Mar 1, 2015; Published: Mar 13, 2015

Mathematics Subject Classifications: 05A17, 05A19

\begin{abstract}
Based on the combinatorial proof of Schur's partition theorem given by Bressoud, and the combinatorial proof of Alladi's partition theorem given by Padmavathamma, Raghavendra and Chandrashekara, we obtain a bijective proof of a partition theorem of Alladi, Andrews and Gordon.
\end{abstract}

Keywords: bijection; partition; Schur's partition theorem; Göllnitz's partition theorem; the Alladi-Andrews-Gordon partition theorem

\section{Introduction}

In 1926, Schur [15] proved one of the most profound results in the theory of partitions, which can be stated as follows.

Theorem 1.1 (Schur). The number of partitions of $n$ into distinct parts $\equiv 1,2(\bmod 3)$ is equal to the number of partitions of $n$ into distinct parts $\lambda_{1}>\lambda_{2}>\lambda_{3}>\cdots$ where $\lambda_{i}-\lambda_{i+1} \geqslant 3$ with strict inequality if $\lambda_{i} \equiv 3(\bmod 3)$.

Throughout this paper $x \equiv y(\bmod M)$ means that $x=y+k M$ for a nonnegative integer $k$, where $x \geqslant y$ and $x>0$. Theorem 1.1 is usually called Schur's celebrated partition theorem of 1926. It was extended by Göllnitz [13] in 1967.

Theorem 1.2 (Göllnitz). Let $B(n)$ be the number of partitions of $n$ into distinct parts $\equiv 2,4,5(\bmod 6)$. Let $C(n)$ be the number of partitions of $n$ into distinct parts $\lambda_{1}>\lambda_{2}>$ $\lambda_{3}>\cdots$ where no part equals 1 or 3 , and $\lambda_{i}-\lambda_{i+1} \geqslant 6$ with strict inequality if $\lambda_{i} \equiv 6,7$ or $9(\bmod 6)$. Then $B(n)=C(n)$. 
Theorem 1.2 is one of the most striking extensions of Theorem 1.1. It is not a priori evident that $B(n)=C(n)$. Göllnitz's proof is quite involved. Andrews gave two simpler proofs of Theorem 1.2, one by generating functions [8], and the other by computer algebra $[9, \S 10]$. Göllnitz [13] also gave the following refinement of Theorem 1.2:

$$
\mathcal{B}(n ; s)=\mathcal{C}(n ; s)
$$

where $\mathcal{B}(n ; s)$ and $\mathcal{C}(n ; s)$ denote, respectively, the number of partitions enumerated by $B(n)$ and $C(n)$ with exactly $s$ parts and the parts $\equiv 6,7$ or $9(\bmod 6)$ are counted twice. Andrews [9] asked for a proof which would offer more insights into the refinement (1.1) of Göllnitz's theorem.

There has been a lot of progress towards this direction, see [1, 5, 14]. The first combinatorial approach to Theorem 1.2 was provided by Alladi [1]. Precisely, Alladi constructed a bijection to prove a three-parameter $q$-identity [1, Eq. (1.2)], which first appeared in [5] and is a deep refinement of Theorem 1.2. However, as mentioned by Alladi [1], his construction can not be used to give a bijection between the sets of partitions of $n$ counted by $B(n)$ and $C(n)$. Padmavathamma, Raghavendra and Chandrashekara [14] presented a bijective proof of another partition theorem due to Alladi [2, Theorem 1], and remarked that their bijection also implies Theorem 1.2. They also noted that their method is very similar in spirit to Bressoud's [11] combinatorial proof of Schur's partition theorem.

By using weighted words introduced by Alladi and Gordon [6, 7], Alladi, Andrews and Gordon [5] obtained a more general partition theorem.

Theorem 1.3 (Alladi-Andrews-Gordon). Let $M \geqslant 6$ and let $r_{1}, r_{2}, r_{3}$ be residues satisfying the following conditions:

$$
0<r_{1}<r_{2}<r_{3}<M \leqslant r_{1}+r_{2} \text { and } r_{1}+M<r_{2}+r_{3} .
$$

Let $B(n ; s)$ denote the number of partitions of $n$ into $s$ distinct parts congruent to $r_{1}, r_{2}$ or $r_{3}(\bmod M)$. Let $C(n ; s)$ denote the number of partitions of $n$ into $s$ distinct parts $\lambda_{1}>\lambda_{2}>\lambda_{3}>\cdots$ such that

(i) each part $\lambda_{i}$ is $\equiv r_{1}, r_{2}, r_{3}, r_{1}+r_{2}, r_{1}+r_{3}$ or $r_{2}+r_{3}(\bmod M)$,

(ii) $\lambda_{i}-\lambda_{i+1} \geqslant M$ with strict inequality if $\lambda_{i} \equiv r_{1}+r_{2}, r_{1}+r_{3}$ or $r_{2}+r_{3}(\bmod M)$,

(iii) the parts $\equiv r_{1}+r_{2}, r_{1}+r_{3}$ or $r_{2}+r_{3}(\bmod M)$ are counted twice.

Then $B(n ; s)=C(n ; s)$.

Clearly, Theorem 1.3 reduces to (1.1) by setting $M=6, r_{1}=2, r_{2}=4$, and $r_{3}=5$. As remarked by Alladi, Andrews and Gordon [5, §1], Theorem 1.3 also generalizes two extensions of (1.1) given by Göllnitz [13, Sätze 4.8 and 4.10]. In fact, Alladi, Andrews and Gordon established a three-parameter key identity [5, Eq. (1.4)] which implies Theorem 1.3. Alladi $[1, \S 6]$ noticed that Jacobi's triple product identity [12, p. 12] can be derived from a special case of this key identity. 
Alladi, Andrews and Berkovich [4] found an interpretation of Theorem 1.3 in terms of partitions into six colored integers, and they obtained a more general theorem on partitions into eleven colored integers. Moreover, they showed that the partition theorem involving eleven colored integers is combinatorially equivalent to a four-parameter keyidentity [4, Eq. (1.7)]. Further studies related to Theorem 1.2 and Theorem 1.3 can be found in Alladi and Andrews [3] and Andrews, Bringmann and K. Mahlburg [10].

The objective of this paper is to provide a bijective proof of Theorem 1.3. Our proof is in the spirit of the combinatorial proof of Alladi's partition theorem [2, Theorem 1] given by Padmavathamma, Raghavendra and Chandrashekara [14].

\section{$2 \quad$ A Bijective Proof of Theorem 1.3}

In this section, we present a bijective proof of Theorem 1.3 . Let $\mathbb{B}(n ; s)$ and $\mathbb{C}(n ; s)$ denote the sets of partitions counted by $B(n ; s)$ and $C(n ; s)$, respectively. We define a map from $\mathbb{B}(n ; s)$ to $\mathbb{C}(n ; s)$, then we show that it is a bijection. We need Lemma 2.1 to transform the congruence condition for integers congruent to $r_{i}+r_{j}$ modulo $M(1 \leqslant i<j \leqslant 3)$ into difference conditions for consecutive integers congruent to $r_{i}$ and $r_{j}$ modulo $M$.

By the conditions in (1.2), we see that

$$
0 \leqslant r_{1}+r_{2}-M<r_{1}+r_{3}-M<r_{1}<r_{2}+r_{3}-M<r_{2}<r_{3}<M .
$$

This implies that $r_{1}, r_{2}, r_{3}, r_{1}+r_{2}, r_{1}+r_{3}$ and $r_{2}+r_{3}$ are distinct modulo $M$. For a partition $\mu$ in $\mathbb{C}(n, s)$, if a part $\mu_{k}$ is congruent to $r_{i}+r_{j}$ modulo $M$, where $1 \leqslant i<j \leqslant 3$, we can represent $\mu_{k}$ as a sum of two positive integers congruent to $r_{i}$ and $r_{j}$ modulo $M$ subject to a difference condition. This property also holds for $\mu_{k}-t M$, where $t$ is an integer such that $\mu_{k}-t M \geqslant r_{i}+r_{j}$.

Lemma 2.1. Let $r_{1}, r_{2}$ and $r_{3}$ be integers satisfying the conditions in (1.2). Let $u$ be a positive integer congruent to $r_{i}+r_{j}$ modulo $M$ and $u \geqslant r_{i}+r_{j}$, where $1 \leqslant i<j \leqslant 3$. Let $w=\left(u-r_{i}-r_{j}\right) / M$. Then for integer $0 \leqslant t \leqslant w, u-t M$ can be uniquely expressed as

$$
u-t M=a_{t}+b_{t},
$$

where $a_{t}$ and $b_{t}$ are positive integers such that

$$
a_{t}, b_{t} \equiv r_{i} \text { or } r_{j}(\bmod M) \text { and } a_{t} \not \equiv b_{t}(\bmod M)
$$

and

$$
0<a_{t}-b_{t}<M
$$

More precisely,

$$
a_{t}=\ell M+r_{j}, \quad b_{t}=\ell M+r_{i},
$$

if $u-t M=2 \ell M+r_{i}+r_{j}$, and

$$
a_{t}=(\ell+1) M+r_{i}, \quad b_{t}=\ell M+r_{j},
$$

if $u-t M=(2 \ell+1) M+r_{i}+r_{j}$, where $\ell$ is a nonnegative integer. 
Proof. Clearly, $u-t M \equiv r_{i}+r_{j}(\bmod M)$ can be deduced from $(2.2)$ and $(2.3)$. To determine $a_{t}$ and $b_{t}$ from (2.2), (2.3) and (2.4), we may represent $u-t M$ by $2 \ell M+r_{i}+r_{j}$ or $(2 \ell+1) M+r_{i}+r_{j}$, where $\ell$ is a nonnegative integer. First consider the case $u-t M=$ $2 \ell M+r_{i}+r_{j}$. There are two possibilities. Subcase 1: $a_{t}=\ell^{\prime} M+r_{i}$ and $b_{t}=\ell^{\prime \prime} M+r_{j}$, where $\ell^{\prime}$ and $\ell^{\prime \prime}$ are nonnegative integers such that $\ell^{\prime}+\ell^{\prime \prime}=2 \ell$. Hence we have

$$
a_{t}-b_{t}=\left(\ell^{\prime}-\ell^{\prime \prime}\right) M+r_{i}-r_{j}=2\left(\ell^{\prime}-\ell\right) M+r_{i}-r_{j} .
$$

Since $0<r_{1}<r_{2}<r_{3}<M$ as given in (1.2), we have

$$
-M<r_{i}-r_{j}<0 \text {. }
$$

Under the condition $a_{t}-b_{t}>0$, it follows from (2.7) and (2.8) that $2\left(\ell^{\prime}-\ell\right) \geqslant 1$. Moreover, since $a_{t}-b_{t}<M$, by $(2.7)$ and $(2.8)$ we get $2\left(\ell^{\prime}-\ell\right) \leqslant 1$. So we deduce that $2\left(\ell^{\prime}-\ell\right)=1$. But this is impossible since $\ell^{\prime}$ and $\ell$ are integers. This means that Subcase 1 cannot happen.

We now consider Subcase 2: $a_{t}=\ell^{\prime} M+r_{j}$ and $b_{t}=\ell^{\prime \prime} M+r_{i}$, where $\ell^{\prime}$ and $\ell^{\prime \prime}$ are nonnegative integers such that $\ell^{\prime}+\ell^{\prime \prime}=2 \ell$. In this case, we have

$$
a_{t}-b_{t}=\left(\ell^{\prime}-\ell^{\prime \prime}\right) M+r_{j}-r_{i}=2\left(\ell^{\prime}-\ell\right) M+r_{j}-r_{i} .
$$

Under the condition $a_{t}-b_{t}>0$, it follows from (2.9) and (2.8) that $2\left(\ell^{\prime}-\ell\right) \geqslant 0$. Moreover, since $a_{t}-b_{t}<M$, by $(2.9)$ and $(2.8)$ we get $2\left(\ell^{\prime}-\ell\right) \leqslant 0$. So we deduce that $\ell^{\prime}=\ell^{\prime \prime}=\ell$, which yields (2.5).

For the case $u-t M=(2 \ell+1) M+r_{i}+r_{j}$, we also consider two subcases. Subcase 1: $a_{t}=\ell^{\prime} M+r_{j}$ and $b_{t}=\ell^{\prime \prime} M+r_{i}$, where $\ell^{\prime}$ and $\ell^{\prime \prime}$ are nonnegative integers such that $\ell^{\prime}+\ell^{\prime \prime}=2 \ell+1$. Subcase 2: $a_{t}=\ell^{\prime} M+r_{i}$ and $b_{t}=\ell^{\prime \prime} M+r_{j}$, where $\ell^{\prime}$ and $\ell^{\prime \prime}$ are nonnegative integers such that $\ell^{\prime}+\ell^{\prime \prime}=2 \ell+1$. In Subcase 1 , there is no solution for $\ell^{\prime}$. In Subcase 2, there is only one solution, that is, $\ell^{\prime}=\ell+1$ and $\ell^{\prime \prime}=\ell$. So we arrive at (2.6). The detailed proof is similar to the argument for the first case and hence it is omitted.

We are now ready to give a bijective proof of Theorem 1.3.

Proof of Theorem 1.3. Define a map $\Phi: \mathbb{B}(n ; s) \longrightarrow \mathbb{C}(n ; s)$ by the following procedure. Let $\lambda=\left(\lambda_{1}, \lambda_{2}, \ldots, \lambda_{s}\right)$ be a partition in $\mathbb{B}(n ; s)$. We aim to construct a partition $\mu$ such that $\mu_{k}-\mu_{k+1} \geqslant M$ with strictly inequality if $\mu_{k} \equiv r_{i}+r_{j}(\bmod M)(1 \leqslant i<j \leqslant$ $3)$. Assume that $\lambda$ has only positive parts. For notational convenience, set $\lambda_{0}=+\infty$. Consider the following two cases.

Case 1: Condition (ii) in Theorem 1.3 holds for all consecutive parts of $\lambda$, that is, for any $1 \leqslant i \leqslant s-1$, we have $\lambda_{i}-\lambda_{i+1} \geqslant M$ with strict inequality if $\lambda_{i}$ is congruent to $r_{1}+r_{2}, r_{1}+r_{3}$ or $r_{2}+r_{3}$ modulo $M$. In this case, we see that $\lambda \in \mathbb{C}(n ; s)$, and we set $\mu=\lambda$.

Case 2: Condition (ii) in Theorem 1.3 does not hold, that is, there exists an integer $i$ such that $\lambda_{i}-\lambda_{i+1}<M$. We choose $i_{1}$ to be the minimum integer subject to this condition. We aim to construct a partition, denoted $\alpha^{(1)}$, such that the condition (ii) holds for the 
first $i_{1}$ parts of $\alpha^{(1)}$. If this can be achieved, then one can iterate this process to find a desired partition in $\mathbb{C}(n, s)$. Here are two subcases.

Subcase 2.1: $\lambda_{i_{1}-1}-\left(\lambda_{i_{1}}+\lambda_{i_{1}+1}\right) \geqslant M$. Let

$$
\alpha^{(1)}=\left(\lambda_{1}, \ldots, \lambda_{i_{1}-2}, \lambda_{i_{1}-1}, \lambda_{i_{1}}+\lambda_{i_{1}+1}, \lambda_{i_{1}+2} \ldots\right) .
$$

It is easily checked that the condition (ii) holds for the first $i_{1}$ parts of $\alpha^{(1)}$, that is, for any $1 \leqslant j \leqslant i_{1}-1$ we have $\alpha_{j}^{(1)}-\alpha_{j+1}^{(1)} \geqslant M$ with strict inequality if $\alpha_{j}^{(1)} \equiv r_{1}+r_{2}, r_{1}+r_{3}$ or $r_{2}+r_{3}(\bmod M)$.

Since $0<\lambda_{i}-\lambda_{i+1}<M$, we get $\lambda_{i} \not \equiv \lambda_{i+1}(\bmod M)$. This means that $\lambda_{i_{1}}+$ $\lambda_{i_{1}+1} \equiv r_{i}+r_{j}(\bmod M)$. So we need also show that $\lambda_{i_{1}}+\lambda_{i_{1}+1}-\lambda_{i_{1}+2}>M$ when $s \geqslant i_{1}+2$. This relation is obvious when $\lambda_{i_{1}}-\lambda_{i_{1}+2} \geqslant M$. We now assume that $\lambda_{i_{1}}-\lambda_{i_{1}+2}<M$. Note that $\lambda_{i_{1}}, \lambda_{i_{1}+1}$ and $\lambda_{i_{1}+2}$ are positive integers congruent to $r_{1}, r_{2}$ or $r_{3}$ modulo $M$. By the condition $0<r_{1}<r_{2}<r_{3}<M$ as given in (1.2) and the assumption $\lambda_{i_{1}}-\lambda_{i_{1}+2}<M$, we see that $\left(\lambda_{i_{1}}, \lambda_{i_{1}+1}, \lambda_{i_{1}+2}\right)$ can be expressed in one of the three forms $\left(\ell M+r_{3}, \ell M+r_{2}, \ell M+r_{1}\right),\left((\ell+1) M+r_{1}, \ell M+r_{3}, \ell M+r_{2}\right)$ and $\left((\ell+1) M+r_{2},(\ell+1) M+r_{1}, \ell M+r_{3}\right)$, where $\ell$ is a nonnegative integer. Using the conditions $0<r_{1}<r_{2}<r_{3}<M, r_{1}+M<r_{2}+r_{3}$ as given in (1.2) and the condition $\lambda_{i_{1}}-\lambda_{i_{1}+2}<M$, one can check that $\lambda_{i_{1}}+\lambda_{i_{1}+1}-\lambda_{i_{1}+2}>M$ holds in any of the above three cases. So we have shown that $\alpha^{(1)}$ is a desired partition in Subcase 2.1.

Subcase 2.2: $\lambda_{i_{1}-1}-\left(\lambda_{i_{1}}+\lambda_{i_{1}+1}\right)<M$. There is a unique integer $1 \leqslant k_{1} \leqslant i_{1}-1$ such that

$$
\lambda_{i_{1}-1-t}-\left(\lambda_{i_{1}}+\lambda_{i_{1}+1}+t M\right)<M
$$

for $0 \leqslant t \leqslant k_{1}-1$, and

$$
\lambda_{i_{1}-1-k_{1}}-\left(\lambda_{i_{1}}+\lambda_{i_{1}+1}+k_{1} M\right) \geqslant M
$$

Let

$$
\alpha^{(1)}=\left(\lambda_{1}, \ldots, \lambda_{i_{1}-1-k_{1}}, \lambda_{i_{1}}+\lambda_{i_{1}+1}+k_{1} M, \lambda_{i_{1}-k_{1}}-M, \ldots, \lambda_{i_{1}-1}-M, \lambda_{i_{1}+2}, \ldots\right) .
$$

As $i_{1}$ is chosen to be the minimum integer $i$ such that $\lambda_{i}-\lambda_{i+1}<M$, for any $1 \leqslant j \leqslant i_{1}-1$, we have $\lambda_{j}-\lambda_{j+1} \geqslant M$. This implies that for $i_{1}-k_{1} \leqslant j \leqslant i_{1}-2,\left(\lambda_{j}-M\right)-\left(\lambda_{j+1}-M\right) \geqslant$ $M$. By (2.11), $\lambda_{i_{1}-1-k_{1}}-\left(\lambda_{i_{1}}+\lambda_{i_{1}+1}+k_{1} M\right) \geqslant M$. To verify the condition (ii) for the first $i_{1}$ parts of $\alpha^{(1)}$, it remains to show that

$$
\left(\lambda_{i_{1}}+\lambda_{i_{1}+1}+k_{1} M\right)-\left(\lambda_{i_{1}-k_{1}}-M\right)>M
$$

since the part $\lambda_{i_{1}}+\lambda_{i_{1}+1}+k_{1} M$ is congruent to $r_{i}+r_{j}$ modulo $M$. Notice that (2.12) can be deduced from (2.10) by setting $t=k_{1}-1$. This completes the proof in Subcase 2.2.

For the partition $\alpha^{(1)}$, if condition (ii) holds for all consecutive parts, then we set $\mu=$ $\alpha^{(1)}$. Otherwise, we can find a minimum integer $i_{2}$ such that $i_{2} \geqslant i_{1}$ and $\alpha_{i_{2}}^{(1)}-\alpha_{i_{2}+1}^{(1)}<M$. Then we may repeat the above process in Case 2. Finally, we obtain a partition $\mu$ for which condition (ii) holds for all consecutive parts. 
We observe that each part of $\mu$ is congruent to $r_{1}, r_{2}, r_{3}, r_{1}+r_{2}, r_{1}+r_{3}$ or $r_{2}+r_{3}$ modulo $M$, and the number of parts of $\lambda$ is equal to the number of parts of $\mu$ if the number of parts congruent to $r_{1}+r_{2}, r_{1}+r_{3}$ or $r_{2}+r_{3}$ modulo $M$ are counted twice. Hence conditions (i) and (iii) in Theorem 1.3 also hold for $\mu$. So we have $\mu \in \mathbb{C}(n ; s)$.

To prove that $\Phi$ is a bijection, we now describe the inverse map $\Phi^{-1}$. Let $\mu=$ $\left(\mu_{1}, \mu_{2}, \ldots, \mu_{v}\right)$ be a partition in $\mathbb{C}(n ; s)$. Assume that $\mu_{1}>\mu_{2}>\cdots>\mu_{v}>0$. We aim to construct a partition $\lambda$ such that $\Phi(\lambda)=\mu$ by transforming the congruence condition for parts congruent to $r_{i}+r_{j}$ modulo $M$ into difference conditions for consecutive parts congruent to $r_{i}$ and $r_{j}$ modulo $M$. For notational convenience, set $\mu_{t+1}=0$ if $\mu_{t}$ is the last positive part of $\mu$. Consider the following two cases.

Case 1: There is no part of $\mu$ that is congruent to $r_{1}+r_{2}, r_{1}+r_{3}$ or $r_{2}+r_{3}$ modulo $M$. In this case, we see that $\mu \in \mathbb{B}(n ; s)$, and we set $\lambda=\mu$.

Case 2: There exists an integer $j$ such that $\mu_{j}$ is congruent to $r_{1}+r_{2}, r_{1}+r_{3}$ or $r_{2}+r_{3}$ modulo $M$. We choose $j_{1}$ to be the maximum integer subject to this condition. Using Lemma 2.1 for $u=\mu_{j_{1}}$ and $t=0$, we get $\mu_{j_{1}}=a_{0}+b_{0}$, where $a_{0}$ and $b_{0}$ are given by (2.5) or (2.6). We can transform $\mu$ into a partition, denoted $\beta^{(1)}$, such that the number of parts congruent to $r_{i}+r_{j}$ modulo $M$ in $\beta^{(1)}$ is one less than the number of parts congruent to $r_{i}+r_{j}$ modulo $M$ in $\mu$. There are two cases.

Case (i): $0 \leqslant \mu_{j_{1}+1}<b_{0}$. Let

$$
\beta^{(1)}=\left(\mu_{1}, \ldots, \mu_{j_{1}-1}, a_{0}, b_{0}, \mu_{j_{1}+1}, \ldots, \mu_{v}\right) .
$$

We claim that $\beta^{(1)}$ is a partition. Let $\beta^{(1)}=\left(\beta_{1}^{(1)}, \beta_{2}^{(1)}, \ldots, \beta_{v+1}^{(1)}\right)$. Since $\mu_{1}>\mu_{2}>\cdots>$ $\mu_{v}>0$, by (2.5) and (2.6), we see that $\mu_{1}>\mu_{2}>\cdots>\mu_{j_{1}-1}>a_{0}>b_{0}>0$ if $\mu_{j_{1}+1}=0$, and $\mu_{1}>\mu_{2}>\cdots>\mu_{j_{1}-1}>a_{0}>b_{0}>\mu_{j_{1}+1}>\cdots>\mu_{v}>0$ if $\mu_{j_{1}+1}>0$. It follows that $\beta_{1}^{(1)}>\beta_{2}^{(1)}>\cdots>\beta_{v+1}^{(1)}>0$.

As $j_{1}$ is the maximum integer such that $\mu_{j_{1}}$ is congruent to $r_{1}+r_{2}, r_{1}+r_{3}$ or $r_{2}+r_{3}$ modulo $M$, for $j_{1} \leqslant t \leqslant v+1$, we have $\beta_{t}^{(1)} \equiv r_{1}, r_{2}$ or $r_{3}(\bmod M)$ since all parts of $\mu$ are congruent to $r_{1}, r_{2}, r_{3}, r_{1}+r_{2}, r_{1}+r_{3}$ or $r_{2}+r_{3}$ modulo $M$. So the number of parts congruent to $r_{i}+r_{j}$ modulo $M$ in $\beta^{(1)}$ is one less than the number of parts congruent to $r_{i}+r_{j}$ modulo $M$ in $\mu$.

Case (ii): $\mu_{j_{1}+1} \geqslant b_{0}$. The following procedure generates a partition $\beta^{(1)}$ from $\mu$. Using Lemma 2.1 for $u=\mu_{j_{1}}$ and $t \geqslant 1$ with $\mu_{j_{1}}-t M \geqslant r_{i}+r_{j}$, we obtain a unique expression $\mu_{j_{1}}-t M=a_{t}+b_{t}$, where $a_{t}$ and $b_{t}$ are given by (2.5) or (2.6). Since $\mu_{j_{1}+1} \geqslant b_{0}$, there is a unique integer $1 \leqslant k_{1} \leqslant v-j_{1}$ such that

$$
\mu_{j_{1}+t+1} \geqslant b_{t}
$$

for $0 \leqslant t \leqslant k_{1}-1$, and

$$
0 \leqslant \mu_{j_{1}+k_{1}+1}<b_{k_{1}} .
$$

Let

$$
\beta^{(1)}=\left(\mu_{1}, \ldots, \mu_{j_{1}-1}, \mu_{j_{1}+1}+M, \ldots, \mu_{j_{1}+k_{1}}+M, a_{k_{1}}, b_{k_{1}}, \mu_{j_{1}+k_{1}+1}, \ldots\right),
$$

and denote $\beta^{(1)}$ by $\left(\beta_{1}^{(1)}, \beta_{2}^{(1)}, \ldots, \beta_{v+1}^{(1)}\right)$. Note that $a_{k_{1}}$ and $b_{k_{1}}$ are congruent to $r_{1}, r_{2}$ or $r_{3}$ modulo $M$. Recall that $j_{1}$ is the maximum integer such that $\mu_{j_{1}}$ is congruent to $r_{1}+r_{2}$, 
$r_{1}+r_{3}$ or $r_{2}+r_{3}$ modulo $M$. Since all parts of $\mu$ are congruent to $r_{1}, r_{2}, r_{3}, r_{1}+r_{2}, r_{1}+r_{3}$ or $r_{2}+r_{3}$ modulo $M$, for $j_{1} \leqslant t \leqslant v+1$, we have $\beta_{t}^{(1)} \equiv r_{1}, r_{2}$ or $r_{3}(\bmod M)$. Hence the number of parts congruent to $r_{i}+r_{j}$ modulo $M$ in $\beta^{(1)}$ is one less than the number of parts congruent to $r_{i}+r_{j}$ modulo $M$ in $\mu$.

It remains to show that $\beta^{(1)}$ is a partition. First, if $j_{1} \geqslant 2$, we need to verify that

$$
\mu_{j_{1}-1}>\mu_{j_{1}+1}+M
$$

Since $\mu=\left(\mu_{1}, \mu_{2}, \ldots, \mu_{v}\right)$ is a partition in $\mathbb{C}(n ; s)$, we have $\mu_{i}-\mu_{i+1} \geqslant M$ for $1 \leqslant i \leqslant v-1$. It follows that

$$
\mu_{j_{1}-1}-\left(\mu_{j_{1}+1}+M\right)=\left(\mu_{j_{1}-1}-\mu_{j_{1}}\right)+\left(\mu_{j_{1}}-\mu_{j_{1}+1}\right)-M>0,
$$

which yields (2.15). Next, we prove that

$$
\mu_{j_{1}+k_{1}}+M>a_{k_{1}} .
$$

We claim that

$$
a_{k_{1}}=b_{k_{1}-1} \text {. }
$$

To derive (2.17), we note that $\mu_{j_{1}}-k_{1} M=a_{k_{1}}+b_{k_{1}}$ and $\mu_{j_{1}}-\left(k_{1}-1\right) M=a_{k_{1}-1}+b_{k_{1}-1}$, where $a_{k_{1}}, b_{k_{1}}, a_{k_{1}-1}$ and $b_{k_{1}-1}$ are given by (2.5) or (2.6). If $\mu_{j_{1}}-k_{1} M$ can be represented by $2 \ell M+r_{i}+r_{j}$, where $\ell$ is a nonnegative integer, then we have $\mu_{j_{1}}-\left(k_{1}-1\right) M=$ $(2 \ell+1) M+r_{i}+r_{j}$. By (2.5) and (2.6) we deduce that

$$
a_{k_{1}}=\ell M+r_{j} \quad \text { and } \quad b_{k_{1}-1}=\ell M+r_{j},
$$

as required. Similarly, it can be shown that (2.17) also holds if $\mu_{j_{1}}-k_{1} M$ can be represented by $(2 \ell+1) M+r_{i}+r_{j}$ for a nonnegative integer $\ell$. So (2.17) is confirmed.

Setting $t=k_{1}-1$ in (2.13) gives

$$
\mu_{j_{1}+k_{1}} \geqslant b_{k_{1}-1}
$$

Combining (2.18) and (2.17), we find that $\mu_{j_{1}+k_{1}} \geqslant a_{k_{1}}$. It follows that

$$
\mu_{j_{1}+k_{1}}+M \geqslant a_{k_{1}}+M>a_{k_{1}} .
$$

This proves (2.16). So we have shown that $\beta^{(1)}$ is a partition. Since $\mu$ is a partition in $\mathbb{C}(n ; s)$, it has distinct parts. Thus we have reached the conclusion that $\beta^{(1)}$ has distinct parts. This completes the proof in Case (ii).

For either case (i) or case (ii), if each part of $\beta^{(1)}$ is congruent to $r_{1}, r_{2}$ or $r_{3}$ modulo $M$, then we set $\lambda=\beta^{(1)}$. Otherwise, we can find a maximum integer $j_{2}$ such that $j_{2}<j_{1}$ and $\beta_{j_{2}}^{(1)}$ is congruent to $r_{1}+r_{2}, r_{1}+r_{3}$ or $r_{2}+r_{3}$ modulo $M$. Then we may iterate the above process until we obtain a partition $\lambda$ with all parts congruent to $r_{1}, r_{2}$ or $r_{3}$ modulo $M$.

Moreover, it can be seen that the number of parts of $\lambda$ is equal to the number of parts of $\mu$ with the convention that the parts congruent to $r_{1}+r_{2}, r_{1}+r_{3}$ or $r_{2}+r_{3}$ modulo $M$ are counted twice. Thus we have $\lambda \in \mathbb{B}(n ; s)$, and so $\Phi$ is surjective. 
Due to the uniqueness of the expression of a positive integer congruent to $r_{i}+r_{j}$ modulo $M$ in Lemma 2.1, we see that every step of $\Phi$ is reversible. Hence $\Phi$ is a bijection between $\mathbb{B}(n, s)$ and $\mathbb{C}(n, s)$. So we have $B(n, s)=C(n, s)$. This completes the proof.

The following example gives an illustration of the map $\Phi$. Let $M=6, r_{1}=2, r_{2}=4$ and $r_{3}=5$, for which the conditions in (1.2) are satisfied. Let

$$
\lambda=(92,70,64,53,52,46,38,35,23,17,4,2),
$$

which is a partition in $\mathbb{B}(496 ; 12)$. In the construction of $\Phi(\lambda)$, the intermediate partitions $\alpha^{(1)}, \alpha^{(2)}$ and $\alpha^{(3)}$ are given below:

$$
\begin{aligned}
& \alpha^{(1)}=(123,86,64,58,46,38,35,23,17,4,2), \\
& \alpha^{(2)}=(123,97,80,58,52,40,23,17,4,2), \\
& \alpha^{(3)}=(123,97,80,58,52,40,23,17,6) .
\end{aligned}
$$

Note that condition (ii) in Theorem 1.3 holds for all consecutive parts of $\alpha^{(3)}$, that is, for $1 \leqslant i \leqslant 8$, we have $\alpha_{i}^{(3)}-\alpha_{i+1}^{(3)} \geqslant M$ with strict inequality if $\alpha_{i}^{(3)}$ is congruent to $r_{1}+r_{2}, r_{1}+r_{3}$ or $r_{2}+r_{3}$ modulo $M$. Moreover, there are only three parts, 123, 97 and 6 , which are congruent to $r_{1}+r_{2}, r_{1}+r_{3}$ or $r_{2}+r_{3}$ modulo $M$, and therefore should be counted twice. Hence

$$
\mu=\alpha^{(3)}=(123,97,80,58,52,40,23,17,6),
$$

which belongs to $\mathbb{C}(496 ; 12)$.

The following example gives an illustration of the inverse map $\Phi^{-1}$. Let $M=6, r_{1}=2$, $r_{2}=4$ and $r_{3}=5$, for which the conditions in (1.2) are satisfied. Let

$$
\mu=(123,97,80,58,52,40,23,17,6),
$$

which is a partition in $\mathbb{C}(496 ; 12)$. The intermediate partitions $\beta^{(1)}, \beta^{(2)}$ and $\beta^{(3)}$ are given below:

$$
\begin{aligned}
& \beta^{(1)}=(123,97,80,58,52,40,23,17,4,2), \\
& \beta^{(2)}=(123,86,64,58,46,38,35,23,17,4,2), \\
& \beta^{(3)}=(92,70,64,53,52,46,38,35,23,17,4,2) .
\end{aligned}
$$

Clearly, all the parts of $\beta^{(3)}$ are congruent to 2,4 or 5 modulo $M$. Hence

$$
\lambda=\beta^{(3)}=(92,70,64,53,52,46,38,35,23,17,4,2),
$$

which belongs to $\mathbb{B}(496 ; 12)$.

\section{Acknowledgments}

I am grateful to Arthur L.B. Yang for valuable suggestions. I also wish to thank the referee for helpful comments. This work was supported by the 973 Project and the National Science Foundation of China. 


\section{References}

[1] K. Alladi, A combinatorial correspondence related to Göllnitz' (big) partition theorem and applications, Trans. Amer. Math. Soc. 349 (1997) 2721-2735.

[2] K. Alladi, A variation on a theme of Slvester - a smoother road to Göllnitz's (Big) theorem, Discrete Math. 196 (1999) 1-11.

[3] K. Alladi and G.E. Andrews, The dual of Göllnitz's (big) partition theorem, Ramanujan J. 36 (2015) 171-201.

[4] K. Alladi, G.E. Andrews and A. Berkovich, A new four parameter $q$-series identity and its partition implications, Invent. Math. 153 (2003) 231-260.

[5] K. Alladi, G.E. Andrews and B. Gordon, Generalizations and refinements of a partition theorem of Göllnitz, J. Reine Angew. Math. 460 (1995) 165-188.

[6] K. Alladi and B. Gordon, Generalizations of Schur's partition thoerem, Manuscr. Math. 79 (1993) 113-126.

[7] K. Alladi and B. Gordon, Schur's partition theorem, companions, refinements and generalizations, Trans. Amer. Math. Soc. 347 (1995) 1591-1608.

[8] G.E. Andrews, On a partition theorem of Göllnitz and related formula, J. Reine Angew. Math. 236 (1969) 37-42.

[9] G.E. Andrews, $q$-Series: Their Development and Application in Analysis, Number Theory, Combinatorics, Physics and Computer Algebra, CBMS Regional Conf. Ser. in Math., 66 AMS, Providence, 1986.

[10] G.E. Andrews, K. Bringmann and K. Mahlburg, Double series representations for Schur's partition function and related identities, J. Combin. Theory Ser. A 132 (2015) $102-119$.

[11] D.M. Bressoud, A combinatorial proof of Schur's 1926 partition theorem, Proc. Amer. Math. Soc. 79 (1980) 338-340.

[12] G. Gasper and M. Rahman, Basic Hypergeometric Series, Encyclopedia Math. Appl., Vol.35, Cambridge University Press, Cambridge, 1990.

[13] H. Göllnitz, Partitionen mit Differenzenbedingungen, J. Reine Angew. Math. 225 (1967) 154-190.

[14] Padmavathamma, R. Raghavendra and B. M. Chandrashekara, A new bijective proof of a partition theorem of K. Alladi, Discrete Math. 287 (2004) 125-128.

[15] I.J. Schur, Zur Additiven Zahlentheorie, S.-B. Preuss. Akad. Wiss. Phys.-Math. Kl., 1926, 488-495. (Reprinted in I. Schur, Gesammelte Abhandlungen, Vol. 2, Springer Verlag, Berlin, 1973, 43-50.) 